\title{
AVALIAÇÃO FUNCIONAL DE HEMIPARÉTICOS CRÔNICOS SUBMETIDOS A CIRCUITO DE TREINAMENTO NUMA ESTRATÉGIA DE SAÚDE DA FAMÍLIA - RELATO DE CASOS
}

Carla De Oliveira Carletti, Mileide Cristina Stoco De Oliveira, Aline Ferreira Lima Gonçalves, Fernando Zandonadi, Larissa Borba André, Andressa Sampaio Pereira, Katiane Mayara Guerrero, Eliane Ferrari Chagas, Augusto Cesinando De Carvalho.

Universidade Estadual Paulista - UNESP, Presidente Prudente, SP. E-mail: carlacarletti28@yahoo.com.br.

\section{RESUMO}

Objetivo: o objetivo do presente estudo foi avaliar a mobilidade funcional, marcha, equilíbrio e destreza manual de dois hemiparéticos crônicos submetidos à FGCT e a exequibilidade desta terapêutica numa ESF de Presidente Prudente-SP. Metodologia: O trabalho é um relato de casos de indivíduos hemiparéticos crônicos submetidos à FGCT. Os participantes foram avaliados por meio do Timed up and go (TUG), Teste de Velocidade de Caminhada de 10 metros (TV10M), Escala de Equilíbrio de Berg (EEB) e Nine Hole Peg Test (9HPT) antes (AV1) e após (AV2) o período de 5 sessões de intervenção da FGCT. Resultados: Ambos os indivíduos hemiparéticos apresentaram melhora nos valores obtidos no TUG, TV10M, EEB, e 9HPT quando comparados os momentos de AV1 com AV2. Conclusão: O presente estudo demonstrou que a FGCT é exequível e melhorou a mobilidade funcional, marcha, equilíbrio e destreza manual de dois hemiparéticos crônicos em atendimento numa ESF de Presidente Prudente-SP.

Palavras-chave: Acidente Vascular Cerebral; Hemiparesia; Marcha; Exercício; Fisioterapia

FUNCTIONAL EVALUATION OF CHRONIC HEMIPARKTHES SUBMITTED TO A TRAINING CIRCUIT IN A FAMILY HEALTH STRATEGY - CASE REPORT

\begin{abstract}
Objective: The objective of the present study was to evaluate the functional mobility, gait, balance and manual dexterity of two chronic hemiparetic patients submitted to FGCT and the feasibility of this therapy in a FHS of Presidente Prudente-SP. Methods: A report of two cases with chronic hemiparetic individuals evaluated by Timed up and go (TUG), 10-Meter Walk Test (TV10M), Berg Balance Scale (BSE) and Nine Hole Peg Test (9HPT) At the time AV1 and AV2, after the intervention period of 5 sessions of FGCT. Results: Both hemiparetic individuals presented improvement in TUG, TV10M, BSE, and 9HPTO when compared to AV1 and AV2 moments. Conclusion: The present study demonstrated that FGCT is feasible and improved the functional mobility, gait, balance and manual dexterity of two chronic hemiparetic in attendance at an FHS of Presidente Prudente-SP.
\end{abstract}

Keywords: Stroke; Hemiparesis; March; Exercise; Physiotherapy. 


\section{INTRODUÇÃO}

O acidente vascular cerebral (AVC) é considerado a principal causa de incapacidade neurológica no mundo, pois gera impacto em diversas funções humanas ${ }^{1}$. O controle motor encontra-se particularmente afetado após o AVC, sendo a hemiparesia a disfunção motora predominante nesses indivíduos. Além disso, encontram-se também os prejuízos das funções motoras, sensitivas, coordenação motora, de equilíbrio e de marcha, além do déficit cognitivo e de linguagem ${ }^{2,3}$. Todos esses prejuízos interferem no desempenho das atividades de vida diária, ocasionando uma limitação funcional ${ }^{3,4}$.

A implementação de programas de reabilitação logo após a ocorrência do AVC, pode promover uma recuperação funcional mais rápida e mais completa ${ }^{2}$. A fisioterapia é um componente do processo de reabilitação, sendo o Conceito Neuroevolutivo Bobath uma das abordagens mais utilizadas, entretanto outras modalidades e recursos terapêuticos surgem com o intuito de melhorar a capacidade funcional de forma em geral, além de promover uma melhor interação social $^{5,6}$.

A fisioterapia em grupo no formato de circuito de treinamento (FGCT) é uma dessas modalidades, na qual exercícios funcionais específicos são realizados de forma intensiva com foco na repetição e progressão contínua em uma série de estações de exercícios dispostos num formato de circuito dirigido. Estudos demonstraram que a FGCT está associada a um maior grau de recuperação funcional em diversas atividades e ainda a uma melhor satisfação no momento da alta da reabilitação do que a fisioterapia convencional individual. Há evidências crescentes de que o treinamento de circuitos é eficaz para melhorar a funcionalidade de pacientes com sequela de AVC na fase crônica ${ }^{5,6}$.

Com a finalidade de reorientar o modelo assistencial do Brasil, criou-se, em 1994, o Programa Saúde da Família, reafirmando os princípios básicos do Sistema Único de Saúde. Posteriormente, a partir da edição da Portaria n. 648/06, que trata da Política Nacional de Atenção básica, passou a ser denominado Estratégia de Saúde da Família (ESF), com o objetivo de representar um modelo assistencial que tem a incumbência de promover acompanhamentos a um número de famílias e atuar com ações voltadas a recuperação, manutenção da saúde, reabilitação de agravos e patologias e toda a assistência necessária para uma melhor atenção básica à saúde ${ }^{7}$.

Diante de um conceito mais abrangente de saúde, associada à qualidade de vida, o âmbito da fisioterapia vem expandindo-se. Assim, gradativamente o fisioterapeuta se integra à atenção básica, ampliando seu espectro de atuação para prevenção de doenças e promoção da saúde ${ }^{8}$. Nesta direção, o Programa de Residência em Fisioterapia da FCT-UNESP, integra a equipe de Saúde das Unidades de Estratégia de Saúde Família e vem desenvolvendo programas de atividade física, atendendo pessoas com hemiparesia e hemiplegia nestes locais.

Dessa forma, o objetivo do presente estudo foi avaliar a mobilidade funcional, marcha, equilíbrio e destreza manual de dois hemiparéticos crônicos submetidos à FGCT e exequibilidade desta terapêutica numa ESF de Presidente Prudente-SP.

\section{METODOLOGIA}

Este trabalho é um relato clínico com uma amostra constituída por indivíduos com diagnóstico clínico de AVC participantes do tratamento de fisioterapia oferecido por uma ESF de Presidente Prudente/SP.

Os critérios de inclusão do estudo foram: indivíduos com hemiparesia decorrente ao AVC com tempo de lesão $\geq$ há 12 meses; marcha com ou sem auxílio de órtese ou meio auxiliar de locomoção e habilidade para realizar teste de caminhada. Já os critérios de exclusão do estudo foram: dupla hemiparesia, tempo de lesão inferior a 12 meses, presença de déficits cognitivos avaliados pelo Mini-exame do Estado Mental (ponto de corte para indivíduos analfabetos 20 e 
para indivíduos com diferentes anos de instrução escolar 25-29), afasia sensitiva ou condições de saúde adversas, tais como outras doenças neurológicas ou ortopédicas não relacionadas ao AVC.

Os voluntários foram informados sobre os objetivos e procedimentos do estudo e após concordarem com sua participação, assinaram um termo de consentimento livre e esclarecido. O estudo foi aprovado pelo Comitê de Ética da FCT-UNESP (CAAE: 41034914.1.0000.5402).

Os hemiparéticos foram submetidos a uma entrevista individual para coleta de dados pessoais, verificação dos critérios de inclusão, sendo posteriormente realizada avaliação inicial (AV1) utilizando testes motores funcionais 1- Timed up and go (TUG) ${ }^{9}$ para avaliar a mobilidade funcional durante a marcha, 2- Teste de Velocidade de Caminhada de 10 metros $\left(\right.$ TV10M) ${ }^{10}$ para avaliar a velocidade da marcha, 3- Escala de Equilíbrio de Berg (EEB) ${ }^{11}$ para verificar o equilíbrio funcional e 4 - Nine Hole Peg Test (9HPT) ${ }^{12}$ utilizado para avaliar a destreza manual. Todos os testes foram repetidos após 5 semanas na avaliação final (AV2).

A FGCT ocorreu uma vez por semana no formato de estações, que corresponde a um determinado exercício, previamente montados em espaços diferentes e interligados. Os exercícios têm diferentes níveis de dificuldades, utilizando bastão, cadeira, elástico, barbante, balde, peças de xadrez, banco e garrafa. Cada indivíduo realiza o exercício na velocidade que consegue, porém é estimulado a realizar cada vez mais rápido para que o nível de dificuldade seja aumentado. Antes de iniciar a terapia foi realizada a aferição da pressão arterial. Eles permaneceram por 4 minutos em cada estação e após este tempo, trocaram de estação. Durante a sessão, que tem duração de 60 minutos, eles passaram por todas as estações. Antes de iniciar as estações, os indivíduos realizavam alongamentos globais, realizados de forma ativa, orientados por um fisioterapeuta. As estações e os exercícios realizados durante a FCGT estão apresentados na tabela 1.

Tabela 1: Estações e exercícios realizados durante a FCGT

\begin{tabular}{l|l}
\hline Estação 1 & $\begin{array}{l}\text { Em pé, paciente segura um bastão em suas mãos e tem que acertar um } \\
\text { alvo em formato de círculo, fixado num poste e ao mesmo tempo deve } \\
\text { realizar abdução de quadril; ao retirar o bastão do alvo deve realizar a } \\
\text { adução de quadril. }\end{array}$ \\
\hline Estação 2 & $\begin{array}{l}\text { Em um banco retangular com altura de } 22 \text { centímetros em relação ao } \\
\text { chão o indivíduo deve realizar tríplice flexão com a perna, de modo a } \\
\text { colocar alternadamente os pés sobre o mesmo. }\end{array}$ \\
\hline Estação 3 & $\begin{array}{l}\text { Paciente deve realizar marcha lateral em barbantes fixados em aparelhos } \\
\text { na praça de ginástica, com altura de 11 centímetros em relação ao chão. }\end{array}$ \\
\hline Estação 4 & $\begin{array}{l}\text { Consistiu do paciente levantar-se de uma cadeira, andar o mais rápido } \\
\text { possível a uma distância de três metros, dar a volta numa garrafa e } \\
\text { retornar. }\end{array}$ \\
\hline Estação 5 & $\begin{array}{l}\text { Em pé, paciente realizava flexão de ombro bilateral com auto assistência } \\
\text { utilizando corda elástica fixada num aparelho de ginástica. }\end{array}$ \\
\hline Estação 6 & $\begin{array}{l}\text { Paciente sentado numa cadeira, 10 peças de xadrez colocadas sobre uma } \\
\text { mesa; paciente pegava uma peça de cada vez e as colocava dentro de um } \\
\text { balde; após colocar todas tinha que retirá-las do balde e as colocar em } \\
\text { cima da mesa novamente. }\end{array}$ \\
\hline
\end{tabular}




\section{RESULTADOS}

A amostra foi constituída por dois indivíduos do sexo masculino com idade média de $54 \pm 7,00$ anos, hipertensos, com diagnóstico clínico de AVC isquêmico com média do tempo de lesão de 78 $\pm 42,00$ meses, apresentando hemiparesia espástica à esquerda.

Os resultados individuais dos testes motores funcionais realizados em AV1 e AV2 encontram-se na Tabela 2.

Tabela 2. Resultados dos testes funcionais por indivíduo.

\begin{tabular}{lc|c|c|c|}
\hline & \multicolumn{2}{c|}{ Indivíduo 1 } & \multicolumn{2}{c|}{ Indivíduo 2 } \\
\cline { 2 - 5 } & AV1 & AV2 & AV1 & AV2 \\
\hline TUG (s) & 10,77 & 9,38 & 16,45 & 15,67 \\
\hline TV10M (m/s) & 1,51 & 1,11 & 0,78 & 0,75 \\
\hline EEB (pontos) & 51 & 55 & 50 & 51 \\
\hline 9HPT_LNP(s) & 23 & 22 & 28 & 25 \\
\hline 9HPT_LP(s) & 369 & 315 & 580 & 284 \\
\hline
\end{tabular}

*Nota: TUG = Timed Up and Go; TV10M = Teste de Velocidade de Marcha de 10 metros; EEB = Escala de Equilíbrio de Berg; 9HPT_LNP = Nine Hole Peg Test_Lado não parético; 9HPT_LP = Nine Hole Peg Test_Lado parético.

\section{DISCUSSÃO}

No presente estudo observou-se melhora da mobilidade funcional, marcha, equilíbrio e destreza manual de ambos os hemiparéticos crônicos após a FGCT. Indivíduos com sequela de AVC encontram-se descondicionados fisicamente, predispostos a um estilo de vida sedentário ao qual dificulta a realização eficaz das atividades de vida diária, aumenta o risco de quedas e contribui para a recorrência de outro $\mathrm{AVC}^{13}$, portanto este trabalho demonstrou que a terapêutica utilizada é viável e exequível em ESF pois é de fácil aplicação e não depende de espaços e materiais sofisticados e tecnologia. A FGCT proporcionou a oportunidade destes hemiparéticos desenvolverem atividades físicas funcionais diminuindo o nível de sedentarismo podendo tornarse mais condicionados.

Dessa forma, a FGCT pode ser utilizada como uma modalidade de recurso fisioterapêutico para intervir nos comprometimentos físicos e na qualidade de vida após a lesão cerebral, além de promover uma visão mais ampla sobre as sequelas, ampliando as possibilidades de tratamento. Esta terapêutica estimula a adesão ao tratamento, pois a presença do parceiro pode amenizar a intolerância ao exercício e consequentemente aumentar sua capacidade aeróbica ${ }^{6}$.

A FGCT demonstrou-se efetiva na melhora (AV2 - AV1) do equilíbrio, marcha e mobilidade funcional de hemiparéticos $\operatorname{crônicos}^{14}$, com um aumento da pontuação na EEB de 7,6 pontos, diminuição do tempo do TUG de 3,8 segundos e aumento dos metros percorridos no Teste de Caminhada de Seis Minutos de 93 metros. Melhoras funcionais semelhantes também foram observadas neste presente estudo mesmo tratando-se de um relato de dois casos.

A função manual está relacionada à capacidade do indivíduo em utilizar uma ou ambas as mãos para manipular objetos de diferentes formas e tamanhos. Essa capacidade é importante para uma melhora interação do indivíduo com o meio e é fundamental para a manutenção de um estilo de vida mais independente. O déficit funcional da mão é uma das sequelas mais significativas no indivíduo hemiparético, visto que a mão tem um papel importante no plano de independência e de autoestima desses indivíduos. Assim, é interessante que ele utilize o membro superior acometido, mesmo que este tenha pouca ou nenhuma preensão ${ }^{15}$. 
A melhora na funcionalidade dos membros superiores dos hemiparéticos deste trabalho demonstra que a FGCT é mais uma terapêutica importante que pode ser utilizada independente e também ser associada a outros tipos de reabilitação para o membro superior parético após AVC como a terapia do espelho, prática de realidade virtual, prática de tarefas repetitivas, biofeedback, estimulação elétrica, treino de força e o método Bobath ${ }^{16,17}$.

Assim, circuitos de treinamento utilizando estações que incluem atividades de destreza manual e/ou coordenação motora de membros superiores devem ser estimulados e pesquisados, para fomentar a literatura que encontra-se escassa neste aspecto. Além disso, circuitos que avaliem a mobilidade funcional, marcha e equilíbrio dos hemiparéticos crônicos utilizando outros delineamentos experimentais com maior tempo de intervenção, amostra e grupo controle devem também ser realizados.

\section{CONCLUSÃO}

O presente estudo demonstrou que a FGCT é exequível e melhorou a mobilidade funcional, marcha, equilíbrio e destreza manual de dois hemiparéticos crônicos em atendimento numa ESF de Presidente Prudente-SP.

\section{REFERÊNCIAS}

1- Lucena EMF, Morais JD, Batista HRL, Mendes LM, Silva SQR, Neves RF et al. A funcionalidade de usuários acometidos por AVE em conformidade com a acessibilidade à reabilitação. ACTA FISIATR. 2011; 18(3): $112-118$.

2- Costa SV, Melo LP, Bezerra VT, Souza FHM. Efeitos da Aplicação do Método Bobath e do Treino em Esteira com Suporte Parcial de Peso na Reabilitação da Marcha Pós-AVC: Uma Revisão Sistemática. $\mathrm{R}$ bras ci Saúde. 2014; 18(2):161-166, https://doi.org/10.4034/RBCS.2014.18.02.11.

3- Lima AP, Cardoso FB. O Efeito de um Programa de Exercícios Físicos sobre a Capacidade Funcional da Marcha Hemiparética de Indivíduos com Acidente Vascular Cerebral. R bras ci Saúde.2014; 18(3):203-208, https://doi.org/10.4034/RBCS.2014.18.03.03.

4- Natalio MA, Nunes GS, Herber V, Michaelsen SM. Relação entre cadência da subida e descida de escada, recuperação motora e equilíbrio em indivíduos com hemiparesia. ACTA FISIATR. 2011; 18(3): $146-150$.

5- English CK, Hillier SL, Stiller KR, Warden-Flood A. Circuit class therapy versus individual physiotherapy sessions during inpatient stroke rehabilitation: a controlled trial. Archives of physical medicine and rehabilitation. ;88(8):955-63, https://doi.org/10.1016/i.apmr.2007.04.010.

6- Carvalho AC, Vanderlei LCM, Bofi TC, Pereira JDAS, Nawa VA. Projeto Hemiplegia - Um modelo de fisioterapia em grupo para hemiplégicos crônicos. Arq Ciênc Saúde 2007;14(3):161-8 161.

7- Biana VL, Teixeira GM, Silva CVL, Bispo EPF, Silva MV. Atuação do fisioterapeuta na saúde da família: desafios e conquistas. J Health Sci Inst. 2014; 32(2):211-18. 
8- Carvalho STRF, Cassia-Bava MCGG. Conhecimentos dos usuários da Estratégia Saúde da Família sobre a fisioterapia. Fisioter Mov. 2011 ;24(4):655-64, https://doi.org/10.1590/S010351502011000400009.

9- 21. Podsiadlo D, Richardson S. The timed "Up \& Go": a test of basic functional mobility for frail elderly persons. J Am Geriatr Soc. 1991;39(2):142-8, https://doi.org/10.1111/j.15325415.1991.tb01616.x.

10-Bowden MG, Balasubramanian CK, Behrman AL, Kautz SA. Validation of a Speed-Based Classification System Using Quantitative Measures of Walking Performance Poststroke. Neurorehab Neural Re. 2008 ;22(6):672-5, https://doi.org/10.1177/1545968308318837

11- Miyamoto ST, Lombardi J, Berg KO, Ramos LR, Natour J. Brazilian version of the Berg balance scale. Braz J Med Biol Res. 2004; 37(1): 1411-1421, https://doi.org/10.1590/S0100$\underline{879 \times 2004000900017}$

12-Correa TV, Paz TSR, Silva AEL, Vieira GP, Leite MAA, Orsini M, Correa CL. Avaliação dos membros superiores na doença de Parkinson: implicações para a Reabilitação Física. Rev Bras Neurol. 2016; 52(2):12-16.

13-Gordon NF, Gulanick M, Costa F, Fletcher G, Franklin BA, Roth EJ et al. Physical activity and exercise recommendations for stroke survivors: An American Heart Association Scientific Statement from the Council on clinical cardiology, subcommittee on exercise, cardiac Rehabilitation, and prevention; the Council on cardiovascular nursing; the council on nutrition, physical activity, and metabolism; and the stroke council. Circulation. 2004;109(1):2031-2041, https://doi.org/10.1161/01.CIR.0000126280.65777.A4.

14- Kim B, Park Y, Seo Y, Park S, Cho H, Moom H et al. Effects of individualized versus group taskoriented circuit training on balance ability and gait endurance in chronic stroke inpatients. 2016; J. Phys. Ther. Sci. 28(1): 1872-1875.

15- Lima KCA, Francisco MM, Freitas PB. Relação entre os desempenhos em diferentes testes frequentemente utilizados na avaliação da função manual. 2012; Fisioter. Mov. 25(3): 517-524, https://doi.org/10.1590/S0103-51502012000300007

16- Nanji LS, Cardoso AT, Costa J, Vaz-Carneiro A. Analysis of the Cochrane Review: Interventions for Improving Upper Limb Function after Stroke. 2014; Cochrane. 28(5):551-553.

17- Thaeme ACF, Pinho PA, Reys B, Rodrigues AC. A reabilitação funcional do membro superior de pacientes espásticos, pós Acidente Vascular Cerebral (AVC). Rev Neurocienc. 2010;18(2):179185 